\title{
BOTANY
}

\section{Field Manual of Michigan Flora}

By E. G. Voss, and A. A. Reznicek. 2013. The University of Michigan Press, c/o Chicago Distribution Center, 11030 South Langley Avenue, Chicago, Illinois 60628 USA. 990 pages, 25.00 CAD, Cloth.

Many field naturalists in eastern North America will be familiar with Edward Voss's classic, three-volume Michigan Flora. But since its publication beginning in 1972, extensive taxonomic changes led many of us to supplement this flora with various updated but often less accessible keys, sometimes photocopied from others, and maybe inconveniently stuffed into a filing cabinet. With the 2012 publication of the Field Manual of Michigan Flora, a single pared-down volume offers a reference that compiles the many changes in nomenclature and plant relationships that have occurred in the last four decades. Over 200 new species for Michigan, a large majority of them non-native, have also been described in this new work.

To achieve the synthesis of three volumes into one while including new species, several important changes have been made. Perhaps most notably, the botanical line drawings of selected species have been removed. This turns out to be less of a limitation than I had anticipated, since I have come to rely upon other sources for most illustrations anyway. Distribution maps for Michigan counties are still present but smaller, and thanks to this more compact form, maps are now physically closer to their corresponding species description. Finally, the concise text is now restricted to identification and distribution, with taxonomic debate referenced rather than treated directly. Like others, I have a fondness for Voss' thorough and often witty introductory text to many genera in the original flora, and will continue to refer to it. However, the advantage of this single volume is undeniable.

As with the preceding Michigan Flora, the stand-out feature of this work continues to be the clarity and high quality of the keys, which are more accessible to beginners and amateurs than any other volume I know. Couplets consist of precise but clear language, almost always with multiple characters. As before, certain keys even permit identification with only vegetative material (e.g. Potamogetonaceae). Practical identification tips for the field botanist are present in many descriptions. Ontario botanists benefit from native-born coauthor Tony Reznicek's extensive knowledge of this area with a number of direct references to Ontario occurrences. The glossary is also substantially more comprehensive, although it would still benefit from a few illustrations for more difficult concepts.

Those already familiar with flora of the area will note many, many nomenclature and taxonomic changes. Some of these follow Flora of North America (FNA) treatments, and some do not. The large number of families and species assignments that have changed since the publication of Michigan Flora are helpfully summarized in a three-page appendix (and take note of
Liliaceae!). The taxonomic concept is relatively conservative in some difficult and highly variable groups (e.g. Rubus, Amelanchier), with quite broadly defined species or complexes.

Organizational changes are mostly welcome. The listing of families by alphabetical order (rather than by taxonomic order) makes the manual more accessible to the non-taxonomist. Following this pattern, genera are also given alphabetically within families, and species within genera. For the most part, this makes the book much easier and faster to use.

I have found one significant exception to this, and that is in the organization of the genus Carex. In general, the Cyperaceae keys are a great strength of these authors, and are among the most-used pages in my copy. However, the alphabetic listing of all Carex species descriptions, rather than by section as in the old Michigan Flora, does not allow for easy comparison of the descriptions of closely related species (e.g. all Ovales). The location of the key for each species is also not referenced in the index. Since many sections and their constituent species have changed since the publication of Michigan Flora, this has left me to flip through over a dozen pages to locate the appropriate section key. The overall result, in my experience, is much less user-friendly than Michigan Flora. Fortunately, this shortcoming appears limited to Carex, since other large families are sufficiently diverse at the generic level that their keys are more compact.

A minor disappointment relates to the identification of non-native species. Given the large number of new additions of non-native species and the increasing attention paid to them in recent years, I would have liked to see the addition of symbols in each key or description, so they could be obvious at-a-glance. As before, this information is available only in the text of each description.

Still, these issues do not detract greatly from the overall utility of this book. As I expected, this has become my main reference for flora in southern Ontario, which has a species assemblage very similar to Michigan. Weighing in at about $3.5 \mathrm{lbs}$, the durable hardcover could even fit into a backpack - at least on shorter trips. Thanks to a foundation grant that supported its publication, it is available at an extremely affordable price. Whether you are a recent beginner ready for more information or an experienced field botanist, this practical work is the new standard identification manual for vascular plants not only in Michigan, but in adjacent areas.

HOLLY J. BiCKERTON

143 Aylmer Avenue, Ottawa, Ontario K1S 2Y1 Canada 